\title{
McKILLOP, A. B., Contours of Canadian Thought. Toronto, University of Toronto Press, 1987. xii-163 p. 13,95 \$.
}

\section{Richard A. Jarrell}

Volume 43, numéro 3, hiver 1990

URI : https://id.erudit.org/iderudit/304822ar

DOI : https://doi.org/10.7202/304822ar

Aller au sommaire du numéro

Éditeur(s)

Institut d'histoire de l'Amérique française

ISSN

0035-2357 (imprimé)

1492-1383 (numérique)

Découvrir la revue

Citer ce compte rendu

Jarrell, R. A. (1990). Compte rendu de [McKILLOP, A. B., Contours of Canadian Thought. Toronto, University of Toronto Press, 1987. xii-163 p. 13,95 \$.] Revue d'histoire de l'Amérique française, 43(3), 416-419.

https://doi.org/10.7202/304822ar d'utilisation que vous pouvez consulter en ligne.

https://apropos.erudit.org/fr/usagers/politique-dutilisation/ 
McKILlOP, A. B., Contours of Canadian Thought. Toronto, University of Toronto Press, 1987. xii-163 p. 13,95\$

Si vous parcouriez une liste des publications récentes parue il y a vingt ans dans la Revue d'histoire de l'Amérique française ou dans la Canadian 
Historical Review, vous seriez frappé par le manque de publications en histoire des idées. L'historiographie anglo-canadienne, en particulier, était encore trop orientée vers l'histoire politique. La génération d'historiens des années soixante et soixante-dix ont élargi leurs horizons en s'intéressant à l'histoire sociale et à celle du travail, à l'histoire économique, à l'histoire militaire, à l'histoire des femmes, ainsi qu'à l'histoire de la science, de la technologie et de la médecine. Toutes ces approches trouvent tribune dans d'imposants colloques offrant un éventail ahurissant de communications. Et pourtant, l'histoire des idées est demeurée un parent pauvre: peu de communications, peu d'articles, peu de livres, et pas assez de considération. A. B. McKillop a été un des rares défenseurs de cet aspect fascinant, bien que trop longtemps négligé, de notre histoire. Comme il le remarque dans ce recueil d'articles, la raison de cette carence n'est pas difficile à percevoir. Les Canadiens, en tant que peuple colonisateur, n'ont jamais produit un Darwin, un Einstein, un Hegel ni un Gide, mais seulement, semble-t-il, de faibles répliques de "vrais» intellectuels. Mais il s'empresse de nous rappeller que nous étions ce que nous étions (et sommes toujours, d'ailleurs) et notre passé intellectuel n'en est pas moins digne de notre intérêt. McKillop et une très petite équipe d'historiens des idées ont essayé de ressusciter notre vie intellectuelle d'autrefois. Certes, elle ne fut pas celle de l'Angleterre ni de la France, mais elle était nôtre, et elle nous renseigne sur ce que nous étions (et sur ce que nous sommes) dans un registre que ni l'histoire sociale ni l'histoire économique ne peuvent atteindre.

Contours of Canadian Thought réunit huit articles publiés entre 1974 et le milieu des années quatre-vingt. Quoique McKillop soit mieux connu pour son livre $A$ Disciplined Intelligence, consacré à la pensée philosophique du dix-neuvième siècle au Canada anglais, ce recueil couvre un territoire un peu plus vaste. Il se limite toutefois aux courants intellectuels du Canada anglais; ceux du Canada français offraient certes quelques points en commun (comme celui de relever le défi de la science), mais leur évolution s'effectuait en parallèle, plutôt qu'en contact avec la pensée canadienne-anglaise.

L'article le plus ancien, «Nationalism, Identity, and Canadian Intellectual History», précise les contours de ce qui a été fait en histoire intellectuelle canadienne en examinant les ouvrages pionniers de Gordon Stewart, de George Rawlyk, de S. F. Wise, de Carl Berger, de Ramsay Cook et de D. G. Jones, depuis la fin des années soixante et le début des années soixante-dix. Tous portèrent leur attention, d'une façon ou d'une autre, sur la continuelle préoccupation des Canadiens anglais à l'endroit de la nationalité ou de l'identité nationale. Étant donné la longue tradition «libérale-nationaliste» des écrits historiques, cette approche est un exercice intimidant pour un historien contemporain. Toutefois, McKillop affirme que l' «Identity is a matter of present existence, not a future pattern to be hammered out on the anvils of the national past; and since today's national existence is tomorrow's national history it becomes a requirement of the historian simply to delineate - neither to condemn nor to celebrate - the contours of that past» (p. 14).

Le filon historiographique est encore exploité dans le deuxième article, «So Little on the Mind», qui jette un peu de lumière sur le développement de l'histoire des idées au Canada anglais en tant que branche spécialisée de la discipline. L'expérience américaine - qui ne fut pas des plus glorieuses nous est servie pour fins de comparaison (et à titre d'illustration?); tandis que 
l'histoire intellectuelle américaine a connu à sa manière grandeur et décadence, l'histoire intellectuelle canadienne n'est guère parvenue, jusqu'ici, à se fondre en une discipline. McKillop suggère d'élargir la recherche de façon à embrasser trois objectifs: «first, to affirm the inherent value - what, for short, we might call the «integrity» of ideas; second, to discern patterns of structure; third, to search for coherence.» (p. 25) L'histoire des idées, selon McKillop, repose sur une vision plus large, que l'on pourrait qualifier, non sans justification, $d$ ' «histoire culturelle».

Les autres articles sont, dans un sens, des études de cas ayant pour objectif la mise en oeuvre de cette approche. L'intérêt principal de McKillop réside dans la pensée des écrivains universitaires de l'époque victorienne. «Science, Humanism, and the Ontario University», écrit en partie comme réplique à un de mes articles, plaide en faveur d'un effort soutenu pour examiner la pensée et les idéaux humanistes des universitaires ontariens. Jusqu'à présent, nous possédons d'excellentes (ainsi que de médiocres) histoires institutionnelles, mais le passé à reconstituer est bien plus riche encore. Sir Daniel Wilson, l'un des penseurs victoriens les plus fascinants du Canada, constitue le sujet principal de deux articles: «Evolution, Ethnology, and Poetic Fancy», qui parle de la réaction de Wilson vis-à-vis de Darwin, et «The Research Ideal and the University of Toronto», qui examine l'adhésion de Wilson à l'idéal plus ancien, humaniste (et britannique) d'une culture d'érudition, et de son désaccord avec l'idéal plus récent, axé sur la recherche, et préconisé par les Allemands et les Américains. Ce qui est intéressant ici, ce ne sont pas seulement les motifs religieux du rejet par Wilson de la vision darwinienne - en cela il n'était guère différent de la plupart des gens de sa génération - mais également la mise à contribution de ses talents littéraires et artistiques dans la formulation de ses opinions. Wilson endossa souvent la science en tant que fondement pour l'instruction technique, mais il accomplit peu de choses pour la promouvoir. Il est toutefois ironique que son plus jeune frère, George, non moins humaniste ni moins poète, ait été nommé le premier professeur de technologie dans le monde anglophone! Le deuxième article doit être lu conjointement avec l'article écrit par P. N. Ross en 1972 sur l'introduction du PhD à Toronto.

La philosophie idéaliste fascine aussi McKillop, et deux de ses articles, «Science, Ethics, and 'Modern Thought'» et «The Idealist Legacy», proposent l'étude de deux hommes qui ont relevé le défi scientifique dans une perspective idéaliste. Le premier article examine les écrits de William Dawson LeSueur, le deuxième discute du philosophe John Watson de l'Université Queen's. LeSueur est parfois mentionné par les historiens de la littérature, et Watson par les historiens de la philosophie; mais presque aucun Canadien ne se souvient d'eux aujourd'hui. Le deuxième article est une bonne synthèse de la pensée de Watson, que McKillop élabore dans son livre A Disciplined Intelligence. Les deux articles insistent sur le fait que les penseurs canadiens du passé n'étaient pas obligés, pour être intéressants, de figurer parmi les «grands de ce monde», préoccupation des Canadiens d'aujourd'hui que l'auteur juge aussi déplaisante que vide de sens.

Le dernier article, «Science, Authority, and the American Empire», franchit plusieurs décennies pour arriver aux années vingt, afin d'examiner comment les intellectuels qui écrivirent dans The Canadian Forum vinrent aux prises avec les défis d'après-guerre d'une science alliée à l'industrie. Il ne 
s'agissait pas tant de savoir comment ni quand adopter la nouvelle technologie, que de réfléchir sur les conséquences de la perte des certitudes morales de l'époque victorienne et sur la véritable portée de la culture et des capitaux américains. Il est à espérer que cet article encouragera d'autres recherches sur l'ajustement des Canadiens aux idéaux industriels et culturels des Américains, car les racines de cette lutte intellectuelle datent de bien avant 1920 .

Contours of Canadian Thought devrait attirer beaucoup de lecteurs non seulement parmi les historiens des idées mais parmi tous ceux qui essayent de comprendre la culture canadienne au dix-neuvième siècle. Quoique les articles soient consacrés, en fait, aux contours de la pensée canadienne-anglaise, l'historien de la pensée canadienne-française y puisera aussi beaucoup d'éléments valables. Par exemple, l'écart entre la réaction de Sir Daniel Wilson à Darwin et celle des professeurs Thomas-Étienne Hamel ou J.-C.-K. Laflamme, de l'Université Laval, est plus petit qu'on ne l'imagine. De plus, les suggestions données par McKillop aux futurs historiens des idées valent aussi bien pour l'une que pour l'autre des deux traditions historiographiques.

Department of Science Studies

Atkinson College

York University

RICHARD A. JARRELL

Traduction: Lalita Lanthier 